\title{
COLREGS-Compliant Target Following for an Unmanned Surface Vehicle in Dynamic Environments
}

\author{
Pranay Agrawal ${ }^{1}$ and John M. Dolan ${ }^{1,2}$
}

\begin{abstract}
This paper presents the autonomous tracking and following of a marine vessel by an Unmanned Surface Vehicle in the presence of dynamic obstacles while following the International Regulations for Preventing Collisions at Sea (COLREGS) rules. The motion prediction for the target vessel is based on Monte-Carlo sampling of dynamically feasible and collisionfree paths with fuzzy weights, leading to a predicted path resembling anthropomorphic driving behavior. This prediction is continuously optimized for a particular target by learning the necessary parameters for a 3-degree-of-freedom model of the vessel and its maneuvering behavior from its path history without any prior knowledge. The path planning for the USV with COLREGS is achieved on a grid-based map in a single stage by incorporating $A *$ path planning with Artificial Terrain Costs for dynamically changing obstacles. Various scenarios for interaction, including multiple civilian and adversarial vessels, are handled by the planner with ease. The effectiveness of the algorithms has been demonstrated both in representative simulations and on-water experiments.
\end{abstract}

\section{INTRODUCTION}

In recent years, the use of autonomous and remotely operated Unmanned Surface Vehicles (USV) has increased substantially for purposes of environmental monitoring, border surveillance, search and rescue, etc. [1]. Their use in surveillance and security in harbor and coastal regions with a focus on handling adversarial situations is gaining importance, but autonomy still poses a big challenge [2]. The aim of this work is to develop an efficient algorithm to follow a marine vessel in a dynamic environment, such as a harbor or coastal region, in the presence of other vessels and moving obstacles. This requires preventing collision with nearby marine vessels by following the maritime "rules of the road" (COLREGS).

The "International Regulations for Prevention of Collision at Sea" (also known as COLREGS) [3], which are required to be followed by all marine vehicles in order to prevent collisions, do not have enough quantitative values to be uniquely formulated into autonomous rules; rule conflict can also occur. Despite this, many attempts have been made to automate these rules using varied approaches such as velocity obstacles (VO) [4, 5], multi-object optimization interval programming [6], fuzzy logic [7] and evolutionary algorithms [8]. A significant work on autonomous motion planning with COLREGS was done by Kuwata et al. [4] by fusing VO with COLREGS rules, introducing hysteresis and safety buffers, and demonstrating its application with

\footnotetext{
${ }^{1}$ The Robotics Institute, Carnegie Mellon University, PA 15213 USA pranayalandrew. $\mathrm{cmu}$. edu and jmdecs. cmu. edu

${ }^{2}$ Department of Electrical and Computer Engineering, Carnegie Mellon University, PA 15213 USA
}

field tests in a single-target scenario. Although their implementation works well for avoiding obstacles in open waters with very little clutter, the local trajectory planning using VO suffers form several drawbacks including failure to generate stable paths in presence of multiple vessels and obstacles in proximity, which we expect to encounter in an harbor, and being constrained to a first-order prediction of the target vessel. A recent work on application of COLREGS rules while following a target was done by Gupta et al. [5] by using a two-stage process, generating the USV's desired trajectory first while ignoring the civilian vessels and then implementing the COLREGS-compliant planner to adjust the surge speed and heading of the USV on the calculated trajectory. Simulations and experiments were performed with a manually controlled target boat and a civilian boat, both broadcasting their GPS positions to the USV, along with virtual obstacles.

The problem of target tracking and motion estimation for a USV has been addressed by many papers under the assumption of constant-bearing motion prediction [9] and under a master-slave system [10]. While they work well in open sea conditions, their application in a cluttered environment is again limited. A survey of recent work in search, pursuit and evasion in mobile robotics can be found here [11]. Some of the state-of-the-art methods for target motion estimation and following in the presence of clutter were developed by Gupta et al. $[12,13]$.

This paper's key contributions are as follows: (1) A singlestage, COLREGS-compliant and dynamically feasible path planner, that generates a trajectory which can be directly navigated over at nearly full surge speed, instead of navigation at lower speed to enforce COLREGS over a non-COLREGScompliant trajectory. (2) Increasing the accuracy of the motion prediction by $12 \%$ over the best of previous methods in representative dynamic obstacle scenarios without a dramatic increase in computation.

\section{PROBLEM FORMULATION}

Given:

1) A fully observable and grid-based dynamic map of the world $\Phi \subseteq \mathbb{R}^{2} \times T$, where $\Phi(x, y, t) \in[0,1]$ represents the traversability of the vessel over the point $[x, y]$ at time $t, 1$ being an impassable obstacle and 0 being the safest.

2) The previous and current states of our USV $X=$ $[x, y] \times[\theta, v, \omega] \times t$, where $[x, y]$ is the position in $\mathbb{R}^{2}$, $\theta$ is the orientation, $v$ is the linear velocity and $\omega$ is the angular velocity at time $t \in[0, T]$. 


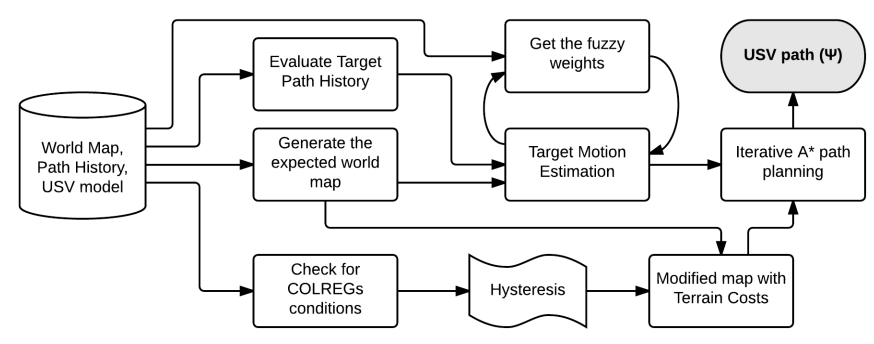

Fig. 1. Flowchart depicting the pipeline for Path Planning

3) The previous and current states of the target vessel $X_{T t}=\left[x_{T t}, y_{T t}\right] \times\left[\theta_{T t}, v_{T t}, \omega_{T t}\right] \times t$ and all the other $n$ vessels in the map $X_{o i}=\left[x_{o i}, y_{o i}\right] \times\left[\theta_{o i}, v_{o i}, \omega_{o i}\right] \times t$, where $t \in[0, T]$ and $i \in[1, n]$.

4) The 3-DOF dynamic model of our USV.

Find:

A path $\Psi=\left[x_{p}, y_{p}, \theta_{p}, t\right]$, consisting of a set of timedependent goal-points for the USV to reach the target vessel within a distance $\varepsilon$ in the least possible time while making sure that it is dynamically feasible, doesn't collide with any obstacle and maintains a safe distance from them, and follows the COLREGS rules with all of the other $n$ vessels $\left(X_{o i}{ }^{\prime} s\right)$ if required.

\section{TARGET FOLLOWING AND MOTION ESTIMATION}

Fig. 1 gives an overview of the proposed three-stage process for the path planning of a USV for the purpose of following another aquatic vessel. The first stage involves the motion estimation of the target vessel over a given time horizon, making use of the target's path history, calculated fuzzy weights and the expected world map. The second involves identifying the COLREGS conditions that apply with the neighboring vessels and modifying the obstacle map, if required, to enforce those rules. The final stage involves iterative $\mathrm{A}^{*}$ search on the 4-dimensional world map for a least-time path to reach close to the target vessel.

Our motion-estimation algorithm to estimate the most probable target-path $X_{T E t}=\left[x_{T E t}, y_{T E t}, \theta_{T E t}, v_{T E t}\right.$, $\left.\omega_{T E t}, t\right]$ is based on the Monte Carlo sampling technique, discussed in [7], wherein we create a large number of probable paths that satisfy our constraints and choose the optimal path from amongst them, while taking into account the target vessel's path history and computing the fuzzy weights for each sampled path based on human driving behavior.

\section{A. Path history and and prospective Move location}

To determine the possible range of motion of the target vessel, we use its observed trajectory to fit a 3-DOF dynamic model for a marine surface vessel to it, as given in Fossen [14]. Observing the path history of the target vessel, we determine its performance envelope comprised of its maximum linear and angular acceleration, maximum linear velocity and maximum angular velocity or minimal turning radius (respectively $\mathrm{a}_{\max }, \alpha_{\max }, v_{\max }, \omega_{\max }$ and $r_{\min }$ ). We

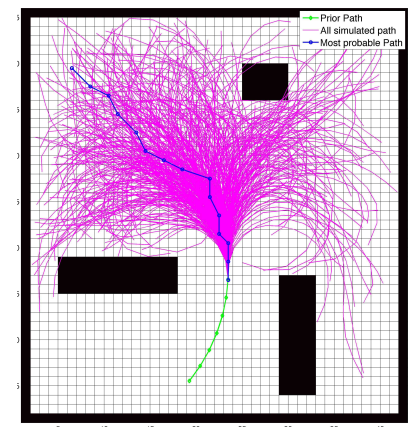

(a) $R_{\text {comp }}=3, V_{\text {comp }}=1$

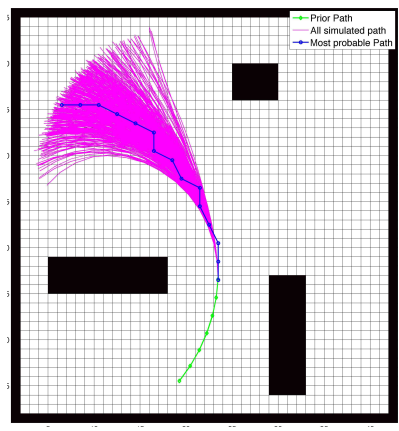

(b) $R_{\text {comp }}=50, V_{\text {comp }}=50$
Fig. 2. Effect of $R_{\text {comp }}$ and $V_{\text {comp }}$ on the spread of sampled Monte Carlo paths

assume that in the future, the target vessel will follow a path in accordance with its 3-DOF model and operate within its observed performance envelope. As this is learnt over time, its accuracy improves the longer we chase the target.

Thus, given either the predicted or the actual state of the target vessel at a time $t, X_{T E t}$, in order to ensure dynamic feasibility, its estimated pose at the next time step, $X_{T E t+1}$, can have a range of values such that $v_{T E(t+1)} \subset$ $\left[\max \left(\left(v_{T E t}-\mathrm{a}_{\max } \times \Delta t\right),-v_{\max }\right), \min \left(\left(v_{T E t}+\mathrm{a}_{\max } \times\right.\right.\right.$ $\left.\Delta t), v_{\max }\right]$ and the angular velocity $\omega_{T E(t+1)} \subset$ $\left[\max \left(\left(\omega_{T E t}-\alpha_{\max } \times \Delta t\right),-\omega_{\max }\right), \min \left(\left(\omega_{T E t}+\alpha_{\max } \times\right.\right.\right.$ $\left.\left.\Delta t), \omega_{\max }\right)\right]$. Also, it should not intersect the obstacle space or other vessels, i.e., $\left.\Phi\left(x_{T E(t+1)}, y_{T E(t+1)}, t+1\right)\right) \neq 1$ and $\left[x_{T E(t+1)}, y_{T E(t+1)}, t+1\right] \not \subset X_{o i}$.

Within this possible range of motions, it is more probable for the vessel to hold its current linear and angular velocities, especially if there are no obstacles present in its expected path, i.e., the path obtained by maintaining the same first-order kinematic states (constant $v_{T} t$ and $\omega_{T} t$ ). At the same time, a high bias towards its current speed and angular velocity results in other possibly safer and more probable paths not being explored. To balance the tradeoff, we also evaluate the target vessel's path history to calculate a desirable radial compression $\left(R_{c o m p}\right)$ and velocity compression $\left(V_{\text {comp }}\right)$, which are measures of bias that we keep respectively towards its current angular and linear velocity while determining its next move. Thus, to determine the $v_{T E(t+1)}$ and $\omega_{T E(t+1)}$ for the next estimated state $X_{T E(t+1)}$, we find normally distributed random values with means $v_{T E t}$ and $\omega_{T E t}$ and standard deviations inversely proportional to $V_{\text {comp }}$ and $R_{\text {comp }}$ respectively, within the constraints discussed earlier.

The procedure to compute a desirable $R_{\text {comp }}$ and $V_{\text {comp }}$ and determine if fuzzy weights should be applied, is given in Algorithm 1. We examine the previous $t_{h}$ seconds of the target vessel's path history and compute the parameters shown below.

1) Velocity consistency $\left(V_{c o n}\right)=$ inverse of the standard deviation of velocity

2) Path straightness $\left(P_{\text {str }}\right)=$ inverse of the weighted mean of the absolute value of angular velocity, recent values 
getting more weight

3) Curvature consistency $\left(C_{c o n}\right)=$ inverse of the standard deviation of the angular velocity

4) Net angular difference $\left(\Delta \theta_{t h}\right)=$ angle moved in the last $t_{h}$ time steps

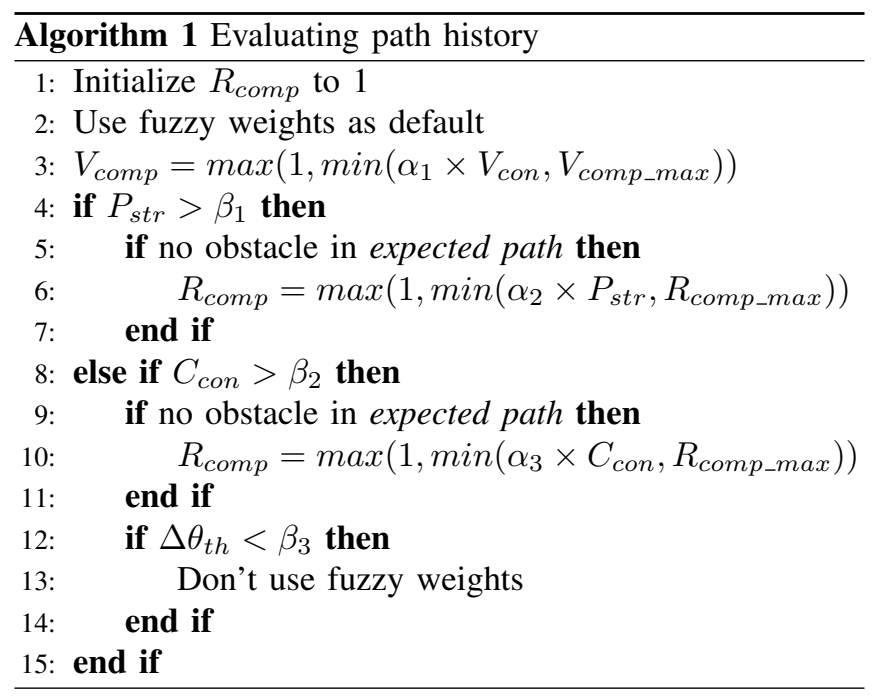

In Algorithm 1, $\alpha_{1}$ controls the velocity compression as a function of velocity consistency, while $\alpha_{2}$ and $\alpha_{3}$ respectively control the radial compression according to the path's straightness and curvature consistency, within the limits of $\left[1, V_{\text {comp_max }}\right]$ and $\left[1, R_{\text {comp_max }}\right] . \beta_{1}, \beta_{2}$ and $\beta_{3}$ respectively control the margins for a path to be classified as a straight path, a path with consistent angular velocity, and a path with high net angular difference. Fig. 2(a) and 2(b) shows the effects of varying $R_{\text {comp }}$ and $V_{\text {comp }}$ on the spread of sampled Monte Carlo paths.

\section{B. Fuzzy Weights}

Along with generating probable and dynamically-feasible paths for the target vessel, in most cases we appoint a fuzzy weight to each of the paths generated through the MonteCarlo sampling so that a path resembling human driving behavior gets higher weight, leading to anthropomorphic motion prediction. The assumptions made here about the human driving are that in a non-evasive target following scenario:

- Coming back very close to the starting location is unlikely

- Suddenly making a large turn and ending up with a very different angle from the original heading is unusual

- Making too many erratic turns is not expected

- Maintaining a safe distance from obstacles is expected

The fuzzy weights $\left(w_{i}\right)$ assigned to each of the generated paths are calculated by a Mamdani's fuzzy system [15] based on the parameters depicted in Fig. 3, developed along the lines of the above assumptions.

\section{Optimal Path Estimation}

Once all paths are generated through the Monte-Carlo sampling and their fuzzy weights calculated, the most prob-

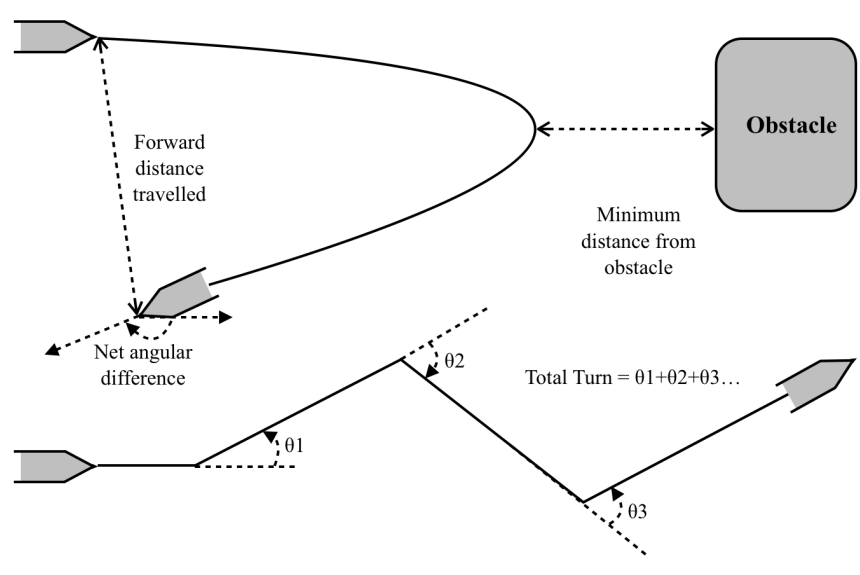

Fig. 3. Parameters for fuzzy weights

able path for the target vessel in the discrete space is calculated for every time interval using Algorithm 2 below. At every time interval, the $\left[x_{T E(t+1)}, y_{T E(t+1)}\right]$ for the next state must be in a region within a maximum radius of $\left(v_{T E t}+\mathrm{a}_{\max } \times \Delta t\right) \times \Delta t$ and a minimum radius of $\left(v_{T E t}-\mathrm{a}_{\max } \times \Delta t\right) \times \Delta t$ from $\left[x_{T E t}, y_{T E t}\right]$. Similarly, $\theta_{T E(t+1)}$ must lie between $\left(\omega_{T E t}+\alpha_{\max } \times \Delta t\right) \times \Delta t$ and $\left(\omega_{T E t}-\alpha_{\max } \times \Delta t\right) \times \Delta t$, thereby leading to additional weights $w_{2}$.

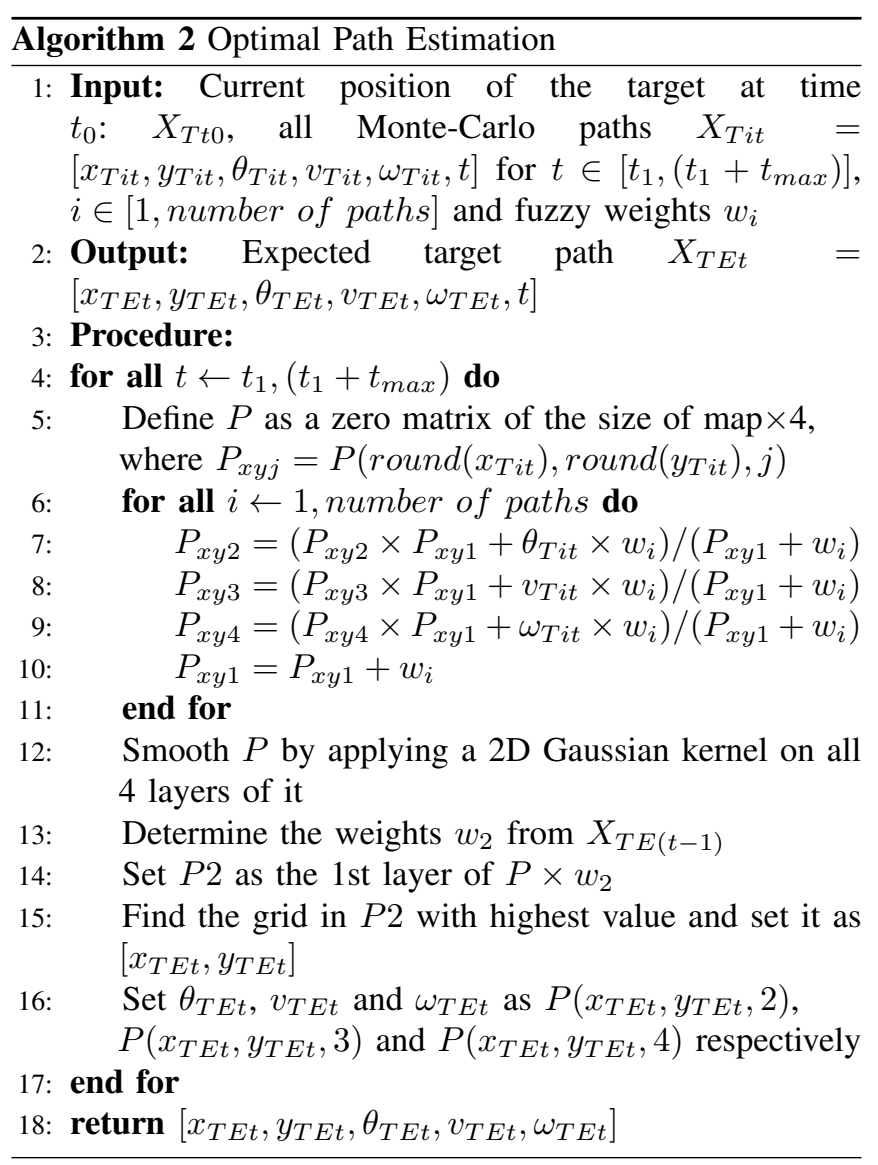




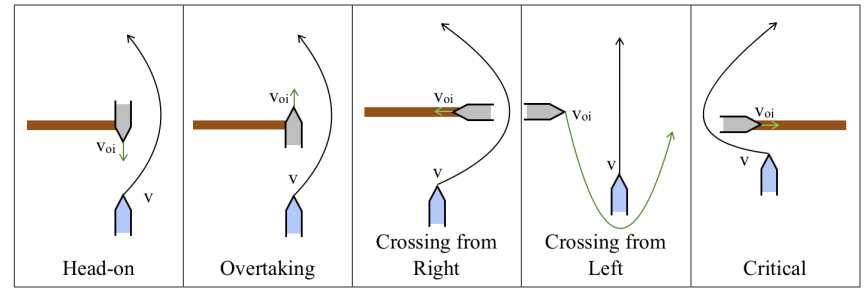

Fig. 4. Figure depicting the possible COLREGS and critical cases, and the appropriate avoidance behavior with Artificial Terrain Costs shown in brown

\section{COLREGS IMPLEMENTATION}

\section{A. COLREGS Cases}

The official COLREGS rulebook [3] specifies a total of 38 rules to be followed in different circumstances for prevention of collision between marine vehicles. Many rules serve only as guidance; in this paper, we address the 4 main cases: Head-on collision, overtaking, crossing from the left and crossing from the right, illustrated in Fig. 4.

To determine if the USV is about to collide with another vessel, we use the Closest Point of Approach (CPA) test [16] with every other vessel in proximity to determine up to a particular time horizon $t_{\max }$, the closest that each vessel is expected to come to the USV $\left(d_{C P A_{-} t_{\max }}\right)$. We apply a COLREGS avoidance maneuver if $d_{C P A_{-} t_{\max }}$ is less than a threshold distance $d_{C O L R E G S}$. If the COLREGS condition applies with a certain vessel, we identify which scenario it belongs to according to their current states and expected relative positions at the CPA, before making the avoidance maneuver. To add stability to the identified rule and ensure that the USV's maneuver is understandable to the neighboring vessels, hysteresis is added. A good way to identify the appropriate rules and to apply hysteresis is given in [9].

If the vessel is already extremely close to the USV $\left(d_{t 0}<d_{\text {critical }}\right)$, the collision is treated as a critical case and the avoidance strategy is executed without consideration to the COLREGS rules with those specific vessels. Such scenarios can arise when the other vessel fails to comply with COLREGS or intentionally comes very close to the USV, as in an adversarial scenario.

\section{B. Implementing the obstacle avoidance maneuver}

To incorporate COLREGS into the A* path-planning, we modify the obstacle map $\Phi \subseteq \mathbb{R}^{2} \times T$ by introducing new obstacles and terrain cost, as shown in Fig. 4. By creating Artificial Terrain Costs in the obstacle map for each vessel $\left(X_{o i}=\left[x_{o i}, y_{o i}\right] \times\left[\theta_{o i}, v_{o i}, \omega_{o i}, t\right]\right)$ through its planning horizon, the USV avoids crossing them from certain directions. The net obstacle map $\Phi$ is calculated by taking the maximum cost from the obstacle map of each vessel $X_{o i}$. The Artificial Terrain Costs are non-lethal, so varying them will change how strongly the COLREGS rules are followed. If the Terrain Costs are set to maximum, the planner would never be able to plan a path over them, which may lead to inability to find a path if there are multiple vessels surrounding the USV.

In the critical case, Artificial Terrain Costs are instead created directly in the direction of the vessel's velocity up to a distance of $v_{o i} / v$ plus a margin to ensure that a collision does not occur. Similarly, Artificial Terrain Costs are also created around other static and dynamic obstacles so that the USV does not get very close to them.

\section{DYNAMIC PATH PLANNING}

The path planning for the USV is done through iterative A* heuristic path planning in 4-dimensional $\mathbb{R}^{2} \times \Theta \times T$ space to find the shortest path in time, while also maintaining a safe distance from the obstacles. Paths are appropriately penalized depending on the number of turns required, how close to an obstacle they get, degree of traversal over high-cost terrain, and their total length. To enforce dynamic feasibility on the computed paths, the next move from the current state is only chosen from a set of options that satisfies the constraints given by the 3-DOF model [14] of our USV.

The paths are iteratively computed from the current pose of the USV $[x, y, \theta, v]$ as the starting point to the successive estimated states of the target vessel $\left[x_{T E t}, y_{T E t}\right]$ as the goal points. At the time the target vessel is expected to reach the goal point, if the USV is expected to be within a distance of $\varepsilon$ from it, the computed path is appointed as the USV's planned path $\left(\Psi=\left[x_{p}, y_{p}, \theta_{p}, t\right]\right)$. Otherwise the goal point is selected as the next estimated state of the target vessel and the same process is repeated until we reach the last estimated state in the planning horizon.

\section{RESULTS}

\section{A. Simulation Results}

To compare the efficiency of the developed motion estimation algorithm with previous methods and to check the implementation of the COLREGS rules with nearby vessels, simulations were carried out in a total of 15 different environments, 5 each with no obstacles, static obstacles and dynamic obstacles. The environments were created to represent a diverse set of real-world scenarios which the USV may encounter in its application, ranging from opensea conditions with high-speed chase to dense harbor-like scenarios with many static and dynamic obstacles. The USV, while following a target vessel executing both evasive and non-evasive manoeuvres, also followed the COLREGS rules with between 1-3 civilian vessels.

Table 1 shows the averaged comparative result for target following with the following motion estimations: (1) Pure Pursuit (PP), where the ownship is steered directly in the direction of the target vessel's current position. (2) Constant Bearing (CB), where the ownship is steered to the CPA given by the vessel's present position, bearing and speed, and ownship's desired chase speed. (3) Simple Monte Carlo (MC) (as presented in [7]). And (4) the proposed Modified MonteCarlo (M-MC) based motion estimation, all with PP as the base-line. In the no obstacles scenarios, the proposed M-MC motion estimation does no better than the $\mathrm{CB}$ estimation, as 


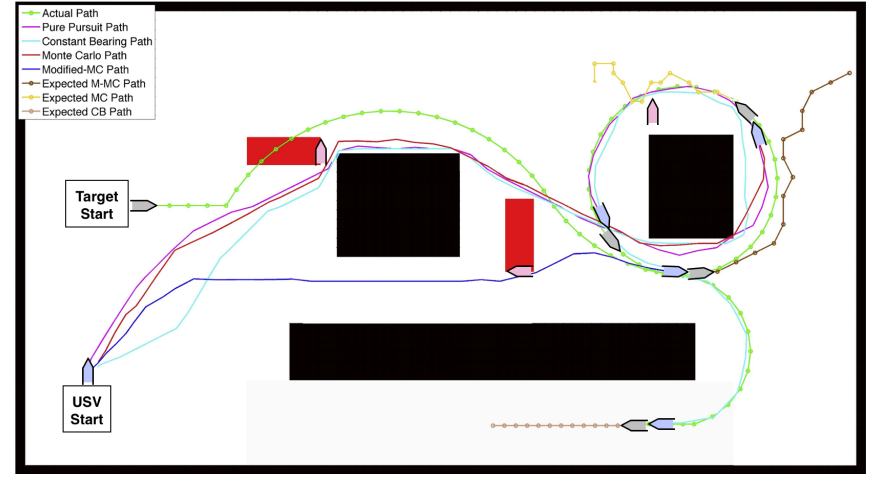

Fig. 5. Figure showing actual target's path, the USV's paths and example instantaneous estimated target paths under different motion estimation types. The black region depicts outer boundary and impassable obstacles. The initial and final positions of the USV and target vessel are shown in blue and gray respectively, the civilian vessel is shown in pink, and the red region depicts the artificial terrain costs created to enforce the COLREGS

the expected path is itself the most probable path of the target vessel. In the cases with static obstacles and dynamic obstacles, under M-MC motion estimation the USV was able to reach the target much faster and in a shorter distance, as it was able to accurately predict the target's path through the obstacles.

TABLE I

COMPARISON BETWEEN DIFFERENT MOTION-ESTIMATION ALGORITHMS

\begin{tabular}{|l|l|r|r|r|}
\hline $\begin{array}{l}\text { Obstacle } \\
\text { Type }\end{array}$ & $\begin{array}{l}\text { Motion Es- } \\
\text { timation }\end{array}$ & $\begin{array}{l}\text { Distance } \\
\text { Travelled }\end{array}$ & $\begin{array}{l}\text { Travel } \\
\text { Time }\end{array}$ & $\begin{array}{l}\text { Computat- } \\
\text { ional Time }\end{array}$ \\
\hline \multirow{2}{*}{ No } & PP & 1 & 1 & 1 \\
Obstacles & CB & 0.8020 & 0.7535 & 5.517 \\
(Free Map) & MC & 0.8237 & 0.8345 & 8.563 \\
& M-MC & 0.8020 & 0.7511 & 12.027 \\
\hline \multirow{3}{*}{ Static } & PP & 1 & 1 & 1 \\
Obstacles & CB & 0.9328 & 0.9517 & 12.519 \\
& MC & 0.8142 & 0.8509 & 15.265 \\
& M-MC & 0.7732 & 0.7725 & 21.163 \\
\hline & PP & 1 & 1 & 1 \\
Dynamic & CB & 0.9023 & 0.8867 & 6.806 \\
Obstacles & MC & 0.8516 & 0.8459 & 11.288 \\
& M-MC & 0.7665 & 0.7333 & 19.508 \\
\hline
\end{tabular}

Fig. 5 shows the target's actual path, USV's paths and estimated target paths for different motion estimation types in a typical static obstacles case. The figure also shows the Artificial Terrain Costs in red to enforce the COLREGS rules. As the USV under M-MC motion-estimation tries to reach the target coming from the top, it is forced to catch it while going to the right of the civilian vessel (in the center) under the "Head-on" COLREGS case. The civilian vessel at the top-left forces the USV coming towards it cross it from its right under the "crossing from Right" case.

The computational time for each cycle, including the path history evaluation, motion estimation, applying COLREGS and iterative $\mathrm{A}^{*}$ path planning was 2.4 seconds for a $100 \times 100 \times 24$ grid on a computer running Matlab on a 2.4 $\mathrm{GHz}$ Intel i5 processor with 500 sampled paths and motion estimation with a projection of 25 units in time. A $10 x+$ reduction in computational time was achieved when porting

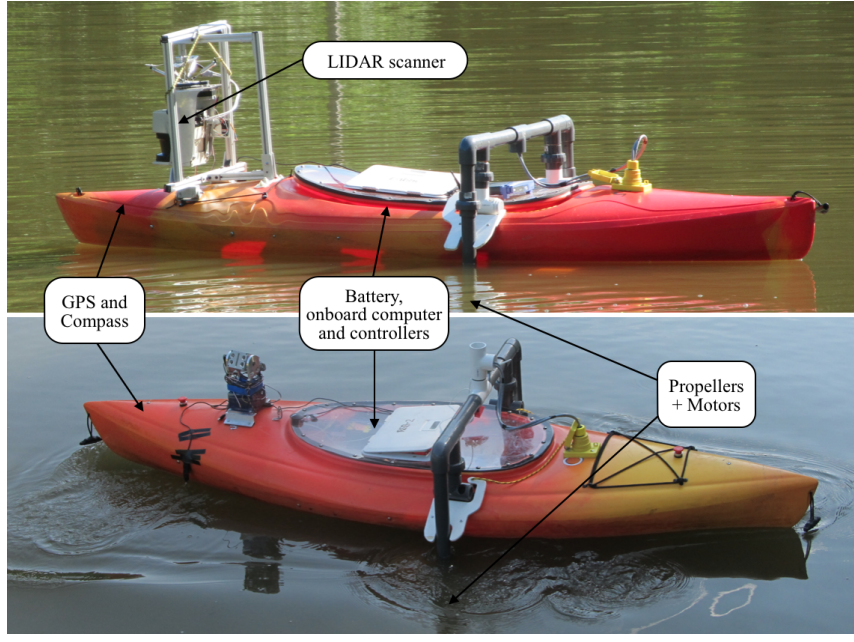

Fig. 6. Autonomous (top) and manually controlled (bottom) kayaks used for the field test

several of the most computationally intensive segments of the code to C++. Assuming a grid size of $4 \mathrm{~m} \times 4 \mathrm{~m} \times 15^{\circ}$, and working within an area of $400 \times 400 \mathrm{~m}$, this loop rate of $4.2 \mathrm{~Hz}$ is fast enough for path planning of a USV moving at a speed of up to $16.8 \mathrm{~m} / \mathrm{s}(60.5 \mathrm{~km} / \mathrm{hr})$.

\section{B. Experimental Results}

The field tests were performed on Panther Hollow Lake in Pittsburgh PA, USA to test the effectiveness of the developed Modified Monte-Carlo target motion estimation in a fully observable map. Both the target-vessel and the USV consisted of two $1.8 \mathrm{~m}$-long kayaks fitted with underwater-scooter motors on either side for propulsion, batteries, computers, compass, GPS, LIDAR scanners for obstacle detection and RF receivers for manual control (Fig. 6). The target-vessel was manually controlled at a speed of $1.7 \mathrm{~m} / \mathrm{s}$ and transmitted its noisy position and heading values to a central computer using an Apache interface. The onboard computer on the autonomous USV was responsible for its path following, obstacle avoidance and sending its sensory values to the central computer, where the high-level computations were performed in Matlab. To carry out the comparative tests, the initial positions of the USV and target vessel, along with the target vessel's path, were fixed for a particular environment. To test the application of COLREGS behavior, virtual obstacles were created with fixed paths $X_{o i}{ }^{\prime} s$.

The results for M-MC motion estimation from the field tests were similar to those acquired in simulation. Fig. 7 shows a comparison of paths taken by the target-vessel and the USV in a typical comparative test with 1 civilian vessel. Under the M-MC motion-estimation, the distance travelled and the time taken to reach the target kayak were 52.2\% and $70.1 \%$ respectively, of that under PP motion-estimation. In the CB case, the distance travelled was $89.1 \%$ and the time taken was $93.0 \%$ that of PP. Fig. 8 shows some screenshots from the field test conducted for M-MC motion estimation. In Fig. 8(a), the USV is in an "Overtaking" COLREGS scenario with the virtual vessel and takes a path from its far left 


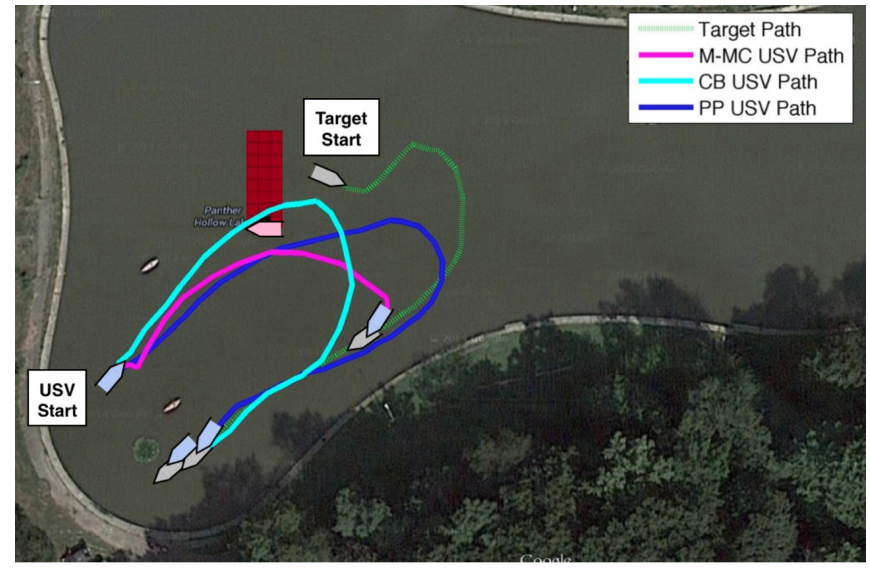

Fig. 7. Compilation of the paths taken by the USV to follow the target vessel in the field test under different target motion-estimations while obeying COLREGS with a civilian vessel (shown in pink). The region with Artificial Terrain Costs for M-MC case is shown in red

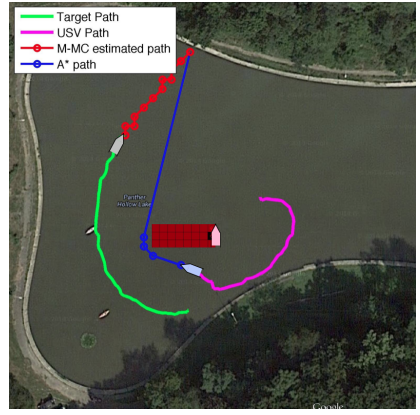

(a) Overtaking

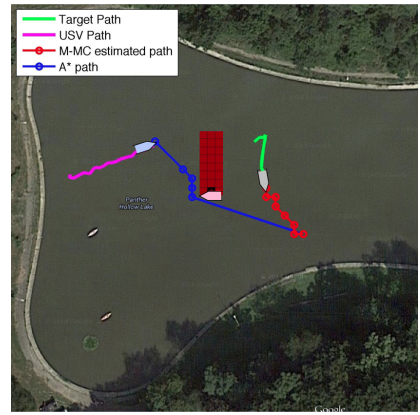

(b) Head-on
Fig. 8. Screenshots from the test showing the COLREGS avoidance manoeuvre for the USV (in blue) with the civilian vessel (in pink) to follow the target vessel (in gray). The regions with Artificial Terrain Costs are shown in red

side, keeping sufficient margin. In Fig. 8(b), the USV is in a "Head-on" collision with the virtual vessel and hence plans a path from its starboard side.

\section{CONCLUSIONS AND FUTURE WORK}

We present accurate and anthropomorphic motion estimation-based autonomous target-following by an Unmanned Surface Vehicle while following the COLEGS rules with nearby marine vessels. The increased accuracy in motion-prediction is achieved by continuously learning the target vessel's navigational behavior from its path history without any prior knowledge and ensuring that the predicted path resembles human driving behavior by the use of fuzzy weights. This integrated single-stage path planning is achieved by fusing the COLREGS rules directly with the dynamic $A^{*}$ heuristic path-planning around dynamic obstacles by imposing Artificial Terrain Costs, while also considering the kinematic and dynamic constraints of the USV. We have demonstrated that this can be achieved robustly and within practical levels of computation, even in situations with multiple civilian vessels and dynamic obstacles, through comparative tests with other motion-estimation methods in simulation and field experiments.

Our future goals are to learn the maneuvering behavior of the target-vessel around obstacles and its evasive maneuver through the use of reinforcement learning. We plan to reduce the computational time for the planner by making use of variable grid size with a higher resolution near the obstacles. We also intend to test our approach on a larger body of water while working at higher speeds and without having the target vessel to broadcast its state.

\section{ACKNOWLEDGMENT}

This research was supported by the National Science Foundation award IIS1124941. Thanks to Weilong Song, Chiyu Dong and Hang Cui for help in performing the field tests.

\section{REFERENCES}

[1] R. J. Yan, S. Pang, H. B. Sun, and Y. J. Pang, "Development and missions of unmanned surface vehicle," Journal of Marine Science and Application 9.4 (2010): pp. 451-457.

[2] S. Campbell, W. Naeem, and G. W. Irwin, "A review on improving the autonomy of unmanned surface vehicles through intelligent collision avoidance manoeuvres," Annual Reviews in Control 36.2 (2012): 267 283.

[3] U.S. Department Of Homeland Security, "U.S. Coast Guard, Navigation Rules," Paradise Cay Publications, 2010.

[4] Y. Kuwata, M. Wolf, D. Zarzhitsky, and T. Huntsberger, "Safe Maritime Navigation with COLREGS Using Velocity Obstacles," Proc. IEEE/RSJ International Conference on Intelligent Robots and Systems (IROS'11), 2011.

[5] P. Svec, B. C. Shah, I. R. Bertaska, J Alvarez, A J. Sinisterra, K. Ellenrieder, M. Dhanak, and S. K. Gupta, "Dynamics-Aware Target Following for an Autonomous Surface Vehicle Operating under COLREGS in Civilian Traffic," Intelligent Robots and Systems (IROS), 2013 IEEE/RSJ.

[6] M. Benjamin, J. Curcio, and P. Newman, "Navigation of Unmanned Marine Vehicles in Accordance with the Rules of the Road," in Proceedings of the IEEE International Conference on Robotics and Automation, 2006.

[7] S.-M. Lee, K.-Y. Kwon, and J. Joh, “A Fuzzy Logic for Autonomous Navigation of Marine Vehicles Satisfying COLREG Guidelines," International Journal of Control Automation and Systems 2 (2004): 171181.

[8] L. P. Perera, J. P. Carvalho, and C. G. Soares, "Autonomous guidance and navigation based on the COLREGS rules and regulations of collision avoidance," in Proceedings of the International Workshop Advanced Ship Design for Pollution Prevention 2009, pp. 205-216.

[9] M. Breivik, V. E. Hovstein, and T. I. Fossen, "Straight-line target tracking for unmanned surface vehicles," Modeling, Identification and Control 29.4 (2008): 131-149.

[10] M. Bibuli, M. Caccia, L. Lapierre, and G. Bruzzone, "Guidance of unmanned surface vehicles: Experiments in vehicle following," Robotics \& Automation Magazine, IEEE, 1999.

[11] T.H. Chung, G.A. Hollinger, and V. Isler, "Search and pursuit-evasion in mobile robotics," Autonomous Robots, pages 1-18, 2011.

[12] P. Svec and S. K. Gupta, "Automated synthesis of action selection policies for unmanned vehicles operating in adverse environments," Autonomous Robots 2012, Volume 32, Issue 2, pp. 149-164.

[13] P. Svec, A. Thakur, B. C. Shah, and S.K. Gupta, "Target following with motion prediction for unmanned surface vehicle operating in cluttered environments," Autonomous Robots, 2013.

[14] T. I. Fossen, "Guidance and control of ocean vehicles," in Wiley, Chicester, England, 1994.

[15] J. H. Lilly, "Mamdani Fuzzy Systems," Fuzzy Control and Identification (2010): 27-45.

[16] S. Arumugam, and C. Jermaine, "Closest-point-of-approach join for moving object histories," Data Engineering, 2006. ICDE'06. Proceedings of the 22nd International Conference on. IEEE, 2006. 